\title{
Plasma Suppression of Large Scale Structure Formation in the Universe
}

\author{
Pisin Chen ${ }^{1,2}$ and Kwang-Chang Lai ${ }^{3}$ \\ ${ }^{1}$ Kavli Institute for Particle Astrophysics and Cosmology, Stanford Linear Accelerator Center, Stanford University, \\ Stanford, California 94309, USA \\ ${ }^{2}$ Department of Physics and Institute of Astrophysics, and Leung Research Center for Cosmology and Particle Astrophysics, \\ National Taiwan University, Taipei 106, Taiwan \\ ${ }^{3}$ Institute of Physics, National Chiao-Tung University, Hsin-Chu 300, Taiwan
}

(Received 12 July 2007; published 4 December 2007)

\begin{abstract}
We point out that during the reionization epoch of the cosmic history, the plasma collective effect among the ordinary matter would suppress the large scale structure formation. The imperfect Debye shielding at finite temperature would induce an electrostatic pressure which, working together with the thermal pressure, would counter the gravitational collapse. As a result, the effective Jeans length, $\tilde{\lambda}_{J}$ is increased by a factor $\tilde{\lambda}_{J} / \lambda_{J}=\sqrt{8 / 5}$, relative to the conventional one. For scales smaller than the effective Jeans scale the plasma would oscillate at the ion-acoustic frequency. The modes that would be influenced by this effect lie roughly in the range $0.5 h \mathrm{Mpc}^{-1}<k$, which corresponds to the Lyman- $\alpha$ forest from the intergalactic medium. We predict that in the linear regime of density-contrast growth, the plasma suppression of the matter power spectrum would approach $1-\left(\Omega_{\mathrm{dm}} / \Omega_{m}\right)^{2} \sim 1-(5 / 6)^{2} \sim 30 \%$.
\end{abstract}

DOI: 10.1103/PhysRevLett.99.231302

PACS numbers: 98.65.Dx, 95.30.Qd, 98.80.Bp

The matter power spectrum of the large-scale structure, along with the cosmic microwave background (CMB) fluctuations, provide important information on the composition and the evolution history of the Universe. Unlike CMB fluctuations, which have been frozen since the recombination time, the matter power spectrum has continued to evolve until now, during which the Universe has been essentially in a reionization, or plasma, state. Plasmas are known to support numerous electron and ion collective oscillation modes. Although in the prerecombination era the Universe was also in a plasma state, the extreme dominance of radiation pressure over that of matter (by a factor $10^{10}$ ) before decoupling renders the contribution of (ep) plasma oscillations totally negligible. In the postdecoupling era, the CMB pressure became negligible. Thus during the reionization epoch [1] one might expect collective plasma effects to exhibit influence on the structure formation.

A static uniform plasma is charge neutral and has no long-range electrostatic potential. But dynamically the inevitable thermal fluctuations of the much more mobile electrons can never perfectly cancel the perturbations of the ions, and that results in a nonvanishing residual electrostatic potential. Such a residual electric force that exerts additional pressure on the ions is noncollisional in nature. When combined with the collision-induced thermal pressure among them, these pressures can support ion-acoustic oscillations, a phenomenon well known in plasma physics [2,3].

In this Letter we point out that the inclusion of such plasma collective effects would result in an increase of the sound speed and therefore an increase of the Jeans scale by a factor $\tilde{\lambda}_{J} / \lambda_{J}=\sqrt{8 / 5}$. The modes that would be influenced by this effect depend on the starting time of reionia- tion and its initial temperature, but should roughly lie in the range $0.5 h \mathrm{Mpc}^{-1}<k$, which corresponds to the region of the Lyman- $\alpha$ forest. This plasma suppression mechanism is effective to both linear and nonlinear regimes of density perturbations. For the sake of tracking the effect, in this Letter we limit our investigation to the linear regime. We predict that the maximum suppression of the power spectrum should approach $\sim 1-\left(\Omega_{\mathrm{dm}} / \Omega_{m}\right)^{2} \sim 1-(5 / 6)^{2} \sim$ $30 \%$.

When the electrostatic potential is included, the standard Einstein-Boltzmann equation in the Newtonian limit is now extended into a Maxwell-Einstein-Boltzmann (MEB) equation. Let us designate the baryon perturbation as $\Delta \equiv \delta \rho_{b} / \bar{\rho}_{b}$, where $\bar{\rho}_{b}$ is the spatially averaged baryon density at a given time $t$. In the electrostatic and the Newtonian limits and with the density perturbation $\Delta \ll$ 1 , the MEB equation reads

$$
\ddot{\Delta}+2 H \dot{\Delta}-\nabla^{2}\left[\frac{\delta P}{\bar{\rho}_{b}}+\frac{e \phi_{e m}}{m_{b}}\right]=4 \pi G \bar{\rho} \Delta,
$$

where the electrostatic potential satisfies the Poisson equation: $\nabla^{2} \phi_{e m}=4 \pi e\left(\delta \rho_{b} / m_{b}-\delta \rho_{e} / m_{e}\right)$. The source of gravity contains both dark matter and baryons, i.e., $\bar{\rho}=$ $\bar{\rho}_{\mathrm{dm}}+\bar{\rho}_{b}$. We assume that the reionization epoch began at a certain time $a^{*}$. Since the baryons fall into the dark matter perturbations after recombination, the baryon fractional overdensity is the same as that of dark matter at the start of reionization, i.e., $\delta \rho_{\mathrm{dm}}\left(a^{*}\right) / \bar{\rho}_{\mathrm{dm}}\left(a^{*}\right)=$ $\delta \rho_{b}\left(a^{*}\right) / \bar{\rho}_{b}\left(a^{*}\right)=\delta \rho\left(a^{*}\right) / \bar{\rho}\left(a^{*}\right) \equiv \Delta^{*}$. After that, dark matter and baryonic matter would still evolve hand-inhand and grow linearly for scales larger than the Jeans scale, but would evolve separately for scales smaller than that. It is this latter situation on which we are focusing for our plasma effect. Thermodynamics governs that 


$$
\frac{\delta P}{\bar{\rho}_{b}}=\frac{\delta P}{\delta \rho_{b}} \frac{\delta \rho_{b}}{\bar{\rho}_{b}}=\frac{\gamma_{b} k_{B} T_{b}}{m_{b}} \Delta .
$$

On the other hand, the balance of forces on electrons requires that the electron number density satisfies the Boltzmann relation: $n_{e}=\bar{n}_{e} \exp \left(e \phi_{e m} / \gamma_{e} k_{B} T_{e}\right) \simeq \bar{n}_{e}[1+$ $\left.e \phi_{e m} / \gamma_{e} k_{B} T_{e}\right]=\rho_{e} / m_{e} \simeq\left[\bar{\rho}_{e}+\delta \rho_{e}\right] / m_{e}$. Inserting this into the Poisson equation, one can derive the electrostatic potential and it is given by

$$
e \phi_{e m} \simeq \frac{4 \pi e^{2} \delta \rho_{e} / m_{e}}{k^{2}+\lambda_{D}^{-2}} \approx \gamma_{e} k_{B} T_{e} \Delta .
$$

The last approximation reflects the fact that our effect relies on the noncollisional interaction among ions through this potential at distances larger than the Debye length, $\lambda_{D} \equiv \sqrt{\gamma_{e} \bar{k}_{B} T_{e} / 4 \pi e^{2} \bar{n}_{e}}$.

In deriving the above potential, we have further assumed that $\delta \rho_{b} / \bar{\rho}_{b} \simeq \delta \rho_{e} / \bar{\rho}_{e}$. This is because globally the fastmoving electrons tend to rapidly readjust themselves to follow the baryon density perturbations. At first sight, it may seem counter-intuitive that this would allow for a nonvanishing electrostatic potential. Because of the inevitable thermal motions of the much lighter and mobile electrons, actually the Debye shielding of the ion density perturbation can never be perfect, and the difference between the local ion and electron densities gives rise to the potential in Eq. (3) in the $k$ space. This, that one assumes $\delta \rho_{e} / \bar{\rho}_{e} \simeq \delta \rho_{b} / \bar{\rho}_{b}$ and $\nabla \cdot E=\nabla^{2} \phi_{e m} \neq 0$ simultaneously, is the so-called plasma approximation, which is a common practice in plasma physics, especially when dealing with ion waves [2].

Inserting Eq. (2) and (3) and Fourier transforming Eq. (1), we arrive at

$$
\ddot{\Delta}_{k}+2 H \dot{\Delta}_{k}+\left(\frac{k}{a}\right)^{2} \tilde{c}_{s}^{2} \Delta_{k}=4 \pi G \bar{\rho} \Delta_{k},
$$

where

$$
\tilde{c}_{s} \equiv\left(\frac{\gamma_{e} k_{B} T_{e}+\gamma_{b} k_{B} T_{b}}{m_{b}}\right)^{1 / 2}=\frac{\omega}{k}
$$

is the "sound speed" of the ion-acoustic waves. The fastmoving electrons are isothermal, so $\gamma_{e}=1$. But for protons, $\gamma_{b}=5 / 3$. In the case where $T_{e}=T_{b}$, the relative change of the sound speed from the ordinary acoustic wave to the ion-acoustic wave is $\sqrt{8 / 5}$. We emphasize that the excitation of the ion-acoustic wave does not rely on the transmission of the Debye-shielded potential across the wavelength, $\sim 1 / k \gg \lambda_{D}$. Analogous to the thermal pressure that arises from local collisions, the electropressure results from the local interaction among ions through their Debye-shielded potentials at distances larger, but of the order of $\lambda_{D}$. Local interaction is a generic property of all pressure waves.

So far the temperature and the onset of the reionization are still unresolved. In the literature (e.g., [4-6]), the reionization temperature ranges from $10^{3} \mathrm{~K}$ to $10^{7} \mathrm{~K}$. As for the onset of reionization, the observation of the GunnPeterson trough in the spectra of high redshift quasars indicates that the reionization process was completed by $z^{*} \approx 6[7,8]$. While the first-year WMAP data determine that $z^{*}=17 \pm 5[9,10]$, more recent WMAP3 finds a lower value of optical depth and hence a lower value for $z^{*}$ [11]. For simplicity and without compromising qualitative features of our effect, we model the reionization as an abrupt transition at a given instant $a^{*}=1 /\left(1+z^{*}\right)$ where the plasma temperature raised instantly to $T_{b}^{*}$ homogeneously throughout the Universe.

To solve Eq. (4) for a given $k$ mode, it is essential to recognize its relationship with the initial and the final Jeans scales at $a^{*}$ and $a$, respectively. Since the Universe expands adiabatically during the matter-dominant epoch, the largescale baryon/plasma temperature satisfies the equation of state, $T_{b} V^{\gamma_{b}-1}=$ const, where $\gamma_{b}=5 / 3$ and $V \propto 1 / \bar{\rho} \propto$ $a^{3}$. Therefore, $T_{b} \propto \tilde{c}_{s}^{2} \propto a^{-2}$. Thus the post-reionization $\left(a>a^{*}\right)$ Jeans scale evolves as

$$
\tilde{k}_{J}(a)=\frac{\sqrt{4 \pi \bar{G} \bar{\rho}(a)}}{\tilde{c}_{s}(a) / a}=\left(\frac{3 \pi}{2} \frac{m_{b} G \bar{\rho}^{*}}{T_{b}^{*}}\right)^{1 / 2} \sqrt{a^{*} a} \geq \tilde{k}_{J}^{*} .
$$

There are three regimes of $k$ modes that concern us. For $k<\tilde{k}_{J}^{*}$, the thermal and the plasma pressures are negligible from the outset. So the baryon perturbation grows linearly in the same way as that of the dark matter: $\Delta_{b} \propto \Delta_{\mathrm{dm}} \propto a$. The decay mode, $\Delta_{b(\mathrm{dm})} \propto a^{-3 / 2}$, fades away in time and can be ignored.

For $k>\tilde{k}_{J}^{*}$, care must be taken regarding $k$ 's further relationship with the Jeans scale at the time of interest. If $k>\tilde{k}_{J}(a)>\tilde{k}_{J}^{*}$, then the combined pressure dominates over the gravity throughout the period $\left(a^{*}, a\right)$. Thus the right-hand side of Eq. (4) is negligible. Changing variable from $t$ to $a$, with $a \propto t^{2 / 3}$ in the matter-dominant era, we find

$$
\frac{d^{2} \Delta_{k}}{d a^{2}}+\frac{3}{2 a} \frac{d \Delta_{k}}{d a}+\left(\frac{k^{2} \tilde{c}_{s}^{2}}{a H_{0}^{2}}\right) \Delta_{k}=0 .
$$

Let us introduce a parameter $\omega_{r}$ which satisfies the relation, $k^{2} \tilde{c}_{s}^{2} / H_{0}^{2} \equiv \omega_{r}^{2} a^{-2}$, such that $\omega_{r}$ is independent of $a$. Since $\tilde{k}_{J} \propto a^{1 / 2}$, we can reexpress it as

$$
\omega_{r}^{2}=\frac{\tilde{c}_{s}^{2} k^{2} a^{2}}{H_{0}^{2}} \simeq \frac{3}{2} \frac{\Omega_{m}}{\Omega_{0}}\left(\frac{k}{\tilde{k}_{J}^{*}}\right)^{2} a^{*} .
$$

It can be shown, through one more change of variable to $x=a^{-2}$, that the solution to Eq. (7) is oscillatory: $\Delta_{k} \propto$ $\exp \left(i 2 \omega_{r} / \sqrt{a}\right)$. Physically, $\omega_{r} / \sqrt{a}$ represents the phase of the ripples of the density contrast in $k$-space due to the ionacoustic oscillation. Such an oscillation begins at the time of reionization, $a^{*}$. Although in reality $\tilde{c}_{s} k$ is the physical ion-acoustic oscillation frequency, $\omega_{r}$ can be viewed as an effective ion-acoustic frequency since it relates to $\tilde{c}_{s} k$ straightforwardly.

Ion-acoustic oscillations are known to suffer Landau damping [2], which was not included in Eq. (7) a priori. 
To include this effect, we invoke a simple empirical formula [2] for the Landau damping dispersion relation that relates the real and the imaginary parts of the ion-acoustic oscillation frequency,

$$
\frac{\omega_{i}}{\omega_{r}} \simeq 1.1\left(\frac{T_{e}}{T_{b}}\right)^{7 / 4} e^{-\left(T_{e} / T_{b}\right)^{2}}, \quad 1 \leq T_{e} / T_{b} \leq 10 .
$$

Since the electron and proton temperatures in the reionization epoch are roughly equal, we find that $\omega_{i} / \omega_{r} \sim 0.4$ in our case. This means that within $\sim 0.4$ of an oscillation, the ion-acoustic wave would be Landau-damped to one $e$-folding of its initial amplitude. Now we replace the $\omega_{r}$ in the exponent of the solution to Eq. (7) by the complex frequency which satisfies the above dispersion relation. Imposing the initial condition at $a^{*}$, we arrive at the perturbation for the $k$-mode whose scale has been below the Jeans scale all the time from $a^{*}$ to $a$, i.e., $k>\tilde{k}_{J}(a)>\tilde{k}_{J}^{*}$,

$$
\Delta_{k} \simeq \Delta_{k}^{*} e^{(-0.8+i 2) \omega_{r} \eta},
$$

where $\eta=\eta\left(a^{*}, a\right) \equiv \sqrt{1 / a^{*}}-\sqrt{1 / a}$.

For the intermediate regime $\tilde{k}_{J}^{*}<k<\tilde{k}_{J}$, the $k$ mode must have exited the Jeans scale at an intermediate time $a_{c}$ : $k=\tilde{k}_{J}\left(a_{c}\right)$, where $a^{*}<a_{c}<a$. Such a mode would oscillate for a period $\Delta a=a_{c}-a^{*}$. Then it would stop the oscillation at $a=a_{c}$ and resume its gravitational collapse during the subsequent interval $a-a_{c}$. Nevertheless, such a resumed growth of perturbation is delayed, because of the ion-acoustic oscillations during the earlier time interval, by the same amount

$$
\Delta a \equiv a_{c}-a^{*}=\left[\left(\frac{k}{\tilde{k}_{J}^{*}}\right)^{2}-1\right] a^{*} .
$$

Thus the evolution of such a mode $\tilde{k}_{J}^{*}<k<\tilde{k}_{J}(a)$ should become

$$
\Delta_{k}=\Delta_{k}^{*}\left(\frac{a-\Delta a}{a^{*}}\right)=\Delta_{k}^{*}\left[\frac{a}{a^{*}}+1-\left(\frac{k}{\tilde{k}_{J}^{*}}\right)^{2}\right] .
$$

Here we have ignored the fact that upon crossing the Jeans scale the $k$ mode may still carry a nonvanishing oscillation amplitude. Such remaining amplitude should be small due to Landau damping.

Our aim is to identify the imprints of this plasma effect in the large-scale structure formation through observations. So we look for its modification to the matter power spectrum [12],

$$
P(k, a)=2 \pi^{2} \delta_{H}^{2} \frac{k^{n}}{H_{0}^{n+3}} T^{2}(k) D^{2}(a) .
$$

Here $\delta_{H}=1.9 \times 10^{-5}$ is the amplitude of primordial perturbations, $H_{0}=100 \mathrm{hm} \mathrm{s}^{-1} \mathrm{Mpc}^{-1}$ the Hubble parameter at present, $T(k)$ the transfer function, and $D(a)$ the growth function. Because of the onset of the plasma effect, the conventional growth function, $D(a)$, in Eq. (13) has to be modified. Let us denote the modified growth function as $\tilde{D}(k, a)$. Clearly, the plasma suppression would only affect the regime where $k>\tilde{k}_{J}^{*}$, which is what we will concentrate on below. To track the plasma effect on the baryons for $a>a^{*}$, we write

$$
\begin{aligned}
\tilde{D}(k, a) & =D_{\mathrm{dm}}(a) \frac{\Omega_{\mathrm{dm}}}{\Omega_{m}}+D_{b}(a) \frac{\Omega_{b}}{\Omega_{m}} \\
& =D_{\mathrm{dm}}\left(a^{*}\right) \frac{a}{a^{*}} \frac{\Omega_{\mathrm{dm}}}{\Omega_{m}}+D_{b}\left(a^{*}\right) \frac{\tilde{\Delta}_{k}}{\Delta_{k}^{*}} \frac{\Omega_{b}}{\Omega_{m}} .
\end{aligned}
$$

Invoking the initial condition, $D_{\mathrm{dm}}\left(a^{*}\right)=D_{b}\left(a^{*}\right)=$ $D\left(a^{*}\right)$, and under the conventional notion of linear growth, $D(a)=D\left(a^{*}\right)\left(a / a^{*}\right)$, we arrive at the relative change of the growth function as

$$
\frac{\tilde{D}(k, a)}{D(a)}=1-\frac{\Omega_{b}}{\Omega_{m}}\left[1-\frac{a^{*}}{a} \frac{\tilde{\Delta}_{k}}{\Delta_{k}^{*}}\right] .
$$

Thus, for a given $k$ mode with $k>\tilde{k}_{J}^{*}$, the plasmasuppressed matter power spectrum relative to that of the conventional approach reads, for $k>\tilde{k}_{J}(a)>\tilde{k}_{J}^{*}$,

$$
\begin{aligned}
\left|\frac{\tilde{D}(k, a)}{D(a)}\right|^{2} \simeq & \left(\frac{\Omega_{\mathrm{dm}}}{\Omega_{m}}\right)^{2}\left\{1+2\left(\frac{\Omega_{b}}{\Omega_{\mathrm{dm}}} \frac{a^{*}}{a}\right) e^{-0.8 \omega_{r} \eta} \cos \left(2 \omega_{r} \eta\right)\right. \\
& \left.+\left(\frac{\Omega_{b}}{\Omega_{\mathrm{dm}}} \frac{a^{*}}{a}\right)^{2} e^{-1.6 \omega_{r} \eta}\right\} .
\end{aligned}
$$

On the other hand we have, for $\tilde{k}_{J}^{*}<k<\tilde{k}_{J}(a)$,

$$
\begin{aligned}
\left|\frac{\tilde{D}(k, a)}{D(a)}\right|^{2} & \simeq\left(\frac{\Omega_{\mathrm{dm}}}{\Omega_{m}}\right)^{2}\left\{1+2\left(\frac{\Omega_{b}}{\Omega_{\mathrm{dm}}} \frac{a^{*}}{a}\right)\left[1+\frac{a}{a^{*}}-\left(\frac{k}{\tilde{k}_{J}^{*}}\right)^{2}\right]\right. \\
& \left.+\left(\frac{\Omega_{b}}{\Omega_{\mathrm{dm}}} \frac{a^{*}}{a}\right)^{2}\left[1+\frac{a}{a^{*}}-\left(\frac{k}{\tilde{k}_{J}^{*}}\right)^{2}\right]^{2}\right\} .
\end{aligned}
$$

To appreciate what this implies in cosmology, let us assume that the reionization occurred at $1+z^{*}=10$, or $a^{*}=0.1$, with the initial plasma temperature $T_{e}^{*} \simeq T_{b}^{*} \sim$ $5 \times 10^{6} \mathrm{~K}$. This corresponds to an ion-acoustic wave velocity of $\tilde{c}_{s}^{*} \sim 7 \times 10^{-4} c$ and $\tilde{k}_{J}^{*} \sim 0.63 \mathrm{~h} \mathrm{Mpc}^{-1}$, and therefore the Jeans length, $\tilde{\lambda}_{J}^{*}=2 \pi / \tilde{k}_{J}^{*} \sim 1.0 h^{-1} \mathrm{Mpc}$.

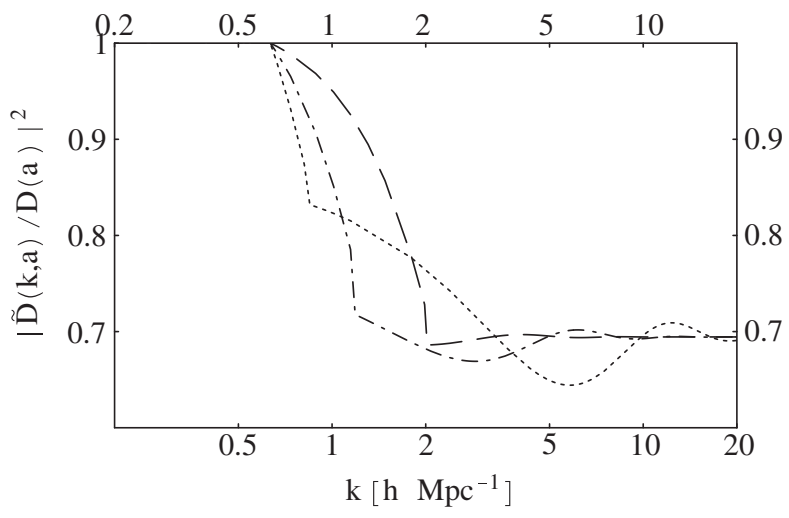

FIG. 1. Suppression of the matter power spectrum. $a^{*}=0.1$ and $T_{b}^{*}=5 \times 10^{6} \mathrm{~K}$. The dotted, dot-dashed, and dashed curves are for $a=1 / 6,1 / 3$, and 1 , respectively. The three regimes of $k$ can be clearly seen. 


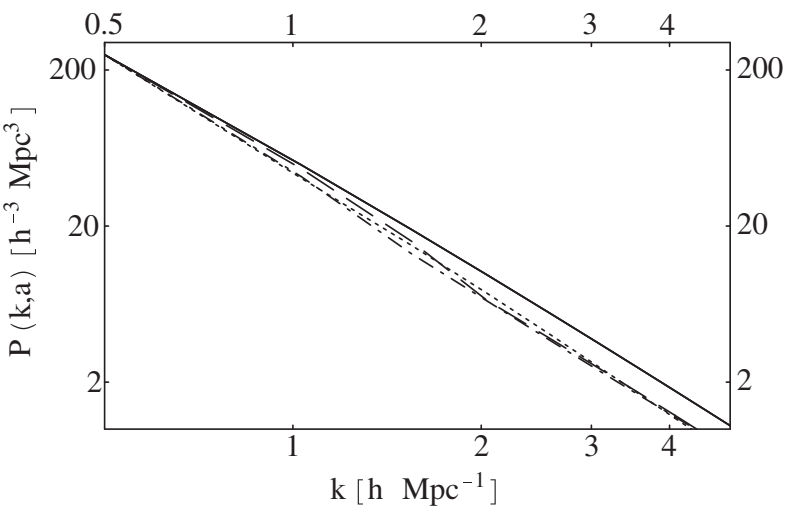

FIG. 2. Matter power spectrum with and without the plasma effect. The conventions are the same as that in Fig. 1.

The Jean's scale at present would be $\tilde{k}_{J}(a=1) \sim$ $2.0 h \mathrm{Mpc}^{-1}$, or $\tilde{\lambda}_{J}(a=1) \sim 3.1 h^{-1} \mathrm{Mpc}$. Figure 1 plots the suppression factor, $|\tilde{D}(k, a) / D(a)|^{2}$, at different times: $a=1 / 6,1 / 3$ and 1 , based on the $\Lambda \mathrm{CDM}$ model with $\Omega_{\Lambda}=0.7, \quad \Omega_{m}=0.3, \Omega_{\mathrm{dm}}=0.25, \quad \Omega_{b}=0.05, a^{*}=$ $0.1, a=1$ and $T_{b}^{*}=5 \times 10^{6} \mathrm{~K}$. The kinks of each curve are associated with the transitions at $\tilde{k}_{J}^{*}$ and $\tilde{k}_{J}\left(a_{c}\right)$, respectively. These kinks are the artifact of our approximation, which should be smeared in reality. The three regimes of $k$, namely, $k<\tilde{k}_{J}^{*}, \tilde{k}_{J}(a=1)>k>\tilde{k}_{J}^{*}$, and $k>\tilde{k}_{J}(a=1)>$ $\tilde{k}_{J}^{*}$, can be readily recognized in this plot. For a mode at $k=\tilde{k}_{J}(a=1)=2.0 \mathrm{~h} \mathrm{Mpc}^{-1}$, for example, it would continue to oscillate until now. Its oscillation "frequency" is $\omega_{r} \sim 0.76$. Thus $2 \omega_{r} \eta \sim 3.3=0.52(2 \pi)$; i.e., it would have oscillated for roughly half a cycle. By then its amplitude would be Landau-damped by more than one $e$ fold. In the last regime, the suppression reaches an asymptotic value of $\sim 70 \%$. Figure 2 compares the conventional and the plasma-suppressed matter power spectra. We have replaced the shape parameter $\Gamma=\Omega_{m} h$ in the BBKS formula [13] for the transfer function by the Eisenstein$\mathrm{Hu}$ [14] rescaled factor. Thus the baryon effects prior to the reionization epoch has been included in the transfer function, which is common to both cases.

By including the long-range residual electrostatic potential, we have shown that the growth of the baryon density contrast during the reionization epoch should be suppressed by the plasma collective effect in the form of ion-acoustic oscillations. Though subject to Landau damping, these oscillations happen to have both the period and the damping time comparable to the Hubble time. Thus there should exist certain imprints of these oscillations in the large-scale structure formation history. In the linear regime of density-contrast growth and the asymptotic limit where the oscillations damp away, the amount of suppression of the matter power spectrum due to the plasma effect reaches $\sim 30 \%$, which is sizable. In principle, the plasma suppression is also effective in the nonlinear regime. It would be interesting to expand our treatment to the nonlinear regime of growth at late times $(a \rightarrow 1)$ and track its impact.

One major effort in modern cosmology is to understand the cosmic composition through the determination of a set of cosmic parameters. In addition to the CMB fluctuations, large-scale structure formation can help further constrain these parameters. In this regard, our predicted plasma suppression of the power spectrum in the Lyman- $\alpha$ forest region may be relevant. We note that the observed power spectrum in the intergalactic medium region indeed appears to be systematically lower than that predicted by the conventional theory [15]. It would be very interesting to confirm this. Aside from this issue, the evolution of the plasma-suppressed power spectrum should provide a unique window to reveal the detail history and dynamics of reionization.

We thank T. Abel, R. Blandford, J.-A. Gu, W. Hu, U.-L. Peng, J. Silk, and R. Wechsler for helpful discussions. K.-C. Lai would like to thank P. Blasi and INAF Osservatorio Astrofisico di Arcetri in Firenze, Italy where the major part of this work was carried out. This work is supported in part by US DOE under Contract No. DE-AC03$76 \mathrm{SF} 00515$.

[1] For a recent review, see, for example, R. Barkana, Science 313, 931 (2006).

[2] Francis F. Chen, Introduction to Plasma Physics and Controlled Fusion, Plasma Physics, Vol. 1 (Plenum, New York, 1984), 2nd ed.

[3] N. A. Krall and A. W. Trivelpiece, Principles of Plasma Physics (McGraw-Hill, New York, 1973).

[4] A. J. Benson, N. Sugiyama, Adi Nusser, and C. G. Lacey, Mon. Not. R. Astron. Soc. 369, 1055 (2006).

[5] M. Tegmark, J. Silk, M. J. Rees, A. Blanchard, T. Abel, and F. Palla, Astrophys. J. 474, 1 (1997).

[6] I. T. Iliev, P. R. Shapiro, E. Scannapieco, G. Mellema, M. Alvarez, A.C. Raga, and Ue-Li Pen, Proceedings IAU Colloquium No. 199, p. 369 (2005).

[7] R. H. Becker et al., Astrophys. J. 122, 2850 (2001).

[8] X. Fan et al., Astrophys. J. 123, 1247 (2002).

[9] A. Kogut et al., Astrophys. J. Suppl. Ser. 148, 161 (2003).

[10] D. N. Spergel et al., Astrophys. J. Suppl. Ser. 148, 175 (2003).

[11] D. N. Spergel et al., Astrophys. J. Suppl. Ser. 170, 377 (2007).

[12] S. Dodelson, Modern Cosmology (Academic, New York, 2003).

[13] J. M. Bardeen, J. R. Bond, N. Kaiser, and A. S. Szalay, Astrophys. J. 15, 304 (1986).

[14] D. J. Eisenstein and W. Hu, Astrophys. J. 496, 605 (1998).

[15] M. Tegmark and M. Zaldarriaga, Phys. Rev. D 66, 103508 (2002). 